EPJ Web of Conferences 49, 12014 (2013)

DOI: $10.1051 /$ epjconf/20134912014

(C) Owned by the authors, published by EDP Sciences, 2013

\title{
Higgs Properties Measurement with the ATLAS Detector at the LHC
}

\author{
Haijun Yang on behalf of the ATLAS Collaboration ${ }^{1, a}$ \\ ${ }^{1}$ Department of Physics, Shanghai Jiao Tong University, 800 Dongchuan Road, Shanghai 200240, China
}

\begin{abstract}
This paper presents an investigation into the properties of the new particle discovered in the search for the Standard Model Higgs boson by the ATLAS experiment at the LHC. The searches in the $H \rightarrow Z Z^{(*)} \rightarrow 4 \ell$, $H \rightarrow \gamma \gamma, H \rightarrow W W^{(*)} \rightarrow \ell v \ell v, H \rightarrow \tau^{+} \tau^{-}$and $H \rightarrow b \bar{b}$ channels include several exclusive final states, which provide sensitivity to the coupling properties of the observed particle through the various production and decay modes. Several benchmarks are considered that are designed to probe different aspects of the SM Higgs boson couplings. No significant deviation from the prediction for a SM Higgs boson is found.
\end{abstract}

\section{Introduction}

The observation of a new particle in the search for the Standard Model (SM) Higgs boson at the LHC, reported by the ATLAS [1] and CMS [2] collaborations, is a milestone in the quest to understand electroweak symmetry breaking. In Ref. [1] the ATLAS collaboration reported the initial estimate for the mass of the particle to be $126.0 \pm 0.4$ (stat) \pm 0.4 (syst) $\mathrm{GeV}$, obtained from the $H \rightarrow \gamma \gamma$ and $H \rightarrow Z Z^{(*)} \rightarrow 4 \ell$ channels. Figure 1 illustrates how this observation was made simultaneously in various SM Higgs boson search channels by showing the best fit value for the global signal strength $\mu$ for a Higgs boson mass hypothesis of $m_{H}=126 \mathrm{GeV}$. The signal strength scales the total number of events from all combinations of production and decay modes relative to their SM values, both for the individual channels and the combination. The signal strength parameter is a convenient observable to test the background-only hypothesis $(\mu=0)$ and the SM Higgs hypothesis $(\mu=1)$. The detailed consistency of the production and decay modes of the new particle with the SM expectations needs to be assessed. This is the object of this paper.

This paper presents the measurements of coupling properties of the observed new particle under several benchmark scenarios. The measured observables are deviations of the couplings from those predicted for a SM Higgs boson. The observed state is assumed to be a CPeven scalar as the Higgs boson of the SM. The results are based on the same analyses and data sets as in Ref. [1], with the same statistical model describing the experimental and theoretical systematic uncertainties. The benchmarks follow the recommendations of the LHC Higgs Cross Section Working Group [3] and references therein.

\footnotetext{
a e-mail: haijun.yang@sjtu.edu.cn
}

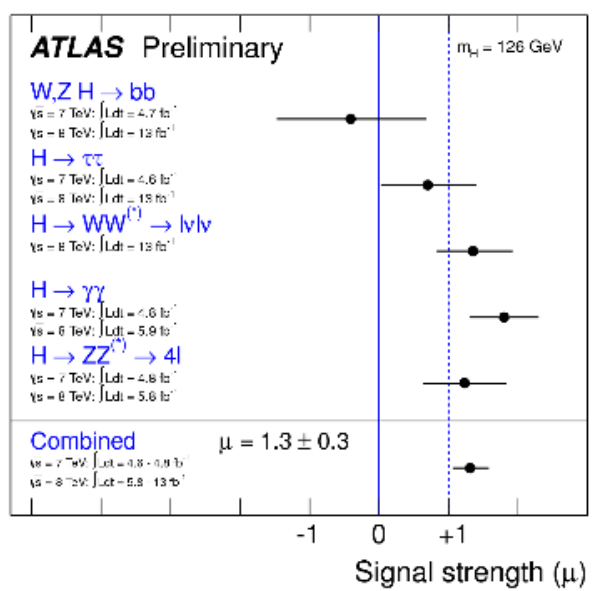

Figure 1. Measurements of the signal strength parameter $\mu$ for $m_{H}=126 \mathrm{GeV}$ for individual channels and their combination (Ref. [8]).

\section{Statistical Procedure}

For each production mode $i$, a signal strength factor $\mu_{i}$ defined by $\mu_{i}=\sigma_{i} / \sigma_{i, S M}$ is introduced, where $\sigma_{i}$ corresponds to the observed cross section and $\sigma_{i, S M}$ to its SM value. Similarly, for each decay final state, $f$, a factor $\mu_{f}=B_{f} / B_{f, S M}$ is introduced, where the $B$ corresponds to decay branching ratios. For each analysis category $(k)$ the number of signal events $\left(n_{\text {signal }}^{k}\right)$ is parametrized as:

$$
n_{\text {signal }}^{k}=\left(\sum_{i} \mu_{i} \times \sigma_{i, S M} \times A_{i}^{k} \times \epsilon_{i f}^{k}\right) \times \mu_{f} \times B_{f, S M} \times \mathcal{L}^{k}
$$

where A represents the detector acceptance, $\epsilon$ the reconstruction efficiency and $\mathcal{L}$ the integrated luminosity. The number of signal events expected from each combination of production and decay is scaled by the corresponding product of $\mu_{i} \mu_{f}$, with no change to the distribution of kinematic or other properties. This parametrization generalizes the dependency of the signal yields on the production cross 
sections and decay branching fractions, allowing for a coherent variation across several channels. The relationship between production and decay in the context of a specific theory or benchmark is achieved via a parametrization of $\mu_{i}, \mu_{f} \rightarrow f(x)$, where the $x$ corresponds to the parameters of the theory or benchmark under consideration.

Given the observed data, the resulting likelihood is a function of a vector of signal strength factors $\mu$, and nuisance parameters $\theta$. Hypothesized values of $\mu$ are tested with a statistic $\Lambda(\mu)$ based on the profile likelihood ratio (Ref. [4])

$$
\Lambda(\mu)=\frac{L(\mu, \hat{\hat{\theta}}(\mu))}{L(\hat{\mu}, \hat{\theta})}
$$

where the signal circumflex denotes the unconditional maximum likelihood estimate of a parameter and the double circumflex sign $(\hat{\hat{\theta}}(\mu))$ denotes the conditional maximum likelihood estimate $(\theta)$ for given fixed values of $\mu$. When the signal strength parameters $\mu$ are reparametrized in terms of $\mu(x)$, the same equation is used for $\Lambda(x)$ with $\mu \rightarrow x$. For all results presented in this paper, the fits are done with a Higgs boson mass hypothesis fixed to its measured value of $m_{H}=126 \mathrm{GeV}$.

\section{Systematic Uncertainties}

The treatment of systematic uncertainties and their correlations is described in detail in Ref. [5]. Systematic uncertainties on observables are handled by introducing nuisance parameters with a probability density function (pdf) associated with the estimate of the correspondent systematic. These nuisance parameters, in particular those representing instrumental uncertainties or background estimates, are often assessed from auxiliary measurements, such as control regions, sidebands, or dedicated calibration measurements. Theoretical uncertainties associated with the branching ratios are small in comparison to both experimental uncertainties and the uncertainty in the production cross sections. Uncertainties in the acceptance due to parton density functions and renormalization and factorization scale choices are included.

\section{Production Signal Strength in Individual Decay Modes}

This section focuses on the measured signal strength in different final states. In the SM, the production cross sections are completely fixed once $m_{H}$ is specified. However, the best fit value for the global signal strength factor $\mu$ does not give any direct information on the relative contributions from different production modes. Furthermore, fixing the ratios of the production cross sections to the ratios predicted by the SM may conceal tension between the data and the SM.

In order to address any tension between the data and the ratios of production cross sections predicted in the SM, the individual channels must separate the signal contribution from various production modes. A test of the SM combining multiple decay modes is complicated by the
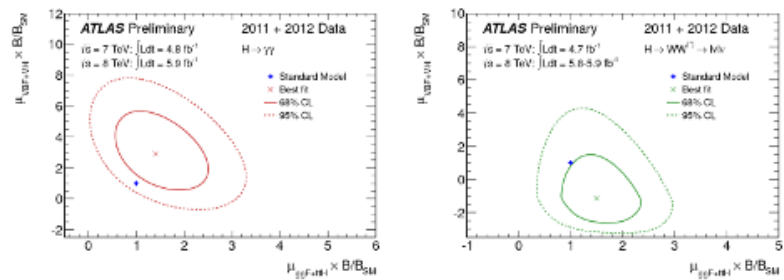

Figure 2. Likelihood contours for the $H \rightarrow \gamma \gamma$ and $H \rightarrow$ $W W^{(*)} \rightarrow \ell v \ell v$ channels in the $\left(\mu_{g g F+t \bar{t} H}, \mu_{V B F+V H}\right)$ plane including the branching ratio factor $B / B_{S M}$. The quantity $\mu_{g g F+t \bar{t} H}\left(\mu_{V B F+V H}\right)$ is a common scale factor for the ggF and $t \bar{t} H$ (VBF and VH) production cross sections. The best fit to the data $(\times)$ and $68 \%$ (solid) and $95 \%$ (dashed) CL contours are also indicated, as well as the SM expectation (+) (Ref. [5]).

fact that the underlying couplings between the Higgs and other particles affect both the production and the decay. Furthermore, parametrization of these effects is subject to a number of assumptions on the presence or absence of new particle states in loop-induced couplings and unobserved decay modes affecting the total width of the Higgs boson.

Since several Higgs boson production modes are available at the LHC, results shown in two dimensional plots require either some $\mu_{i}$ to be fixed or several $\mu_{i}$ to be related. No direct $t \bar{t} H$ production has been observed yet, hence $\mu_{g g H}$ and the very small contribution of $\mu_{t \bar{t} H}$ have been grouped together as they scale dominantly with the $t \bar{t} H$ coupling in the SM and are denoted by the common parameter $\mu_{g q F+t \bar{t} H}$. Similarly, $\mu_{V B F}$ and $\mu_{V H}$ have been grouped together as they scale with the $\mathrm{WH} / \mathrm{ZH}$ gauge coupling in the SM and are denoted by the common parameter $\mu_{V B F+V H}$. The resulting contours for the $H \rightarrow \gamma \gamma$ and $H \rightarrow W W^{(*)} \rightarrow \ell v \ell v$ channels at $m_{H}=126 \mathrm{GeV}$ are shown in Figure 2. For the $H \rightarrow Z Z^{(*)} \rightarrow 4 \ell$ channel only the inclusive analysis has been performed due to the limited statistical power of this channel.

It should be noted that the factors $\mu_{f}$ in different decay modes could have different values. Hence a direct comparison of the results among different final states is not possible. Such comparisons need consistent coupling modifications in the initial state and the final state. It is possible to use their ratio to eliminate the dependence on the branching fraction and illustrate the relative discriminating power between e.g. $g g H+t \bar{t} H$ and $\mathrm{VBF}+\mathrm{VH}$, as well as the compatibility of the measurements in each channel. The likelihood as a function of the ratio $\mu_{V B F+V H} / \mu_{g g F+t \bar{t} H}$ for the $H \rightarrow \gamma \gamma$ and $H \rightarrow W W^{(*)} \rightarrow \ell \nu \ell v$ channels, as well as their combinations are shown in Figure 3.

\section{Measurements of Coupling Properties}

Following the framework and benchmarks as recommended in Ref. [6], measurements of coupling scale factors are implemented using a lowest-order-motivated framework. This framework makes the following assumptions: 


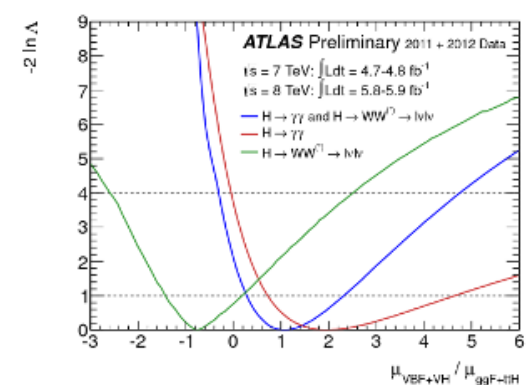

Figure 3. Likelihood curves for $\mu_{V B F+V H} / \mu_{g g F+t \bar{t} H}$ from the $H \rightarrow$ $\gamma \gamma$ and $H \rightarrow W W^{(*)} \rightarrow \ell v \ell v$ channels and their combination (Ref. [5]).

- Only modifications of couplings strenghts, i.e. absolute values of couplings, are taken into account, the observed state is assumed to be a CP-even scalar as in the SM.

- The signals observed in the different search channels originate from a single narrow resonance with a mass of $126 \mathrm{GeV}$. The case of several, possibly overlapping, resonances in this mass region is not considered.

- The width of the Higgs boson with a mass of 126 $\mathrm{GeV}$ is assumed to be negligible. Hence the product $\sigma \times B R(i i \rightarrow H \rightarrow f f)$ can be decomposed in the following way for all channels:

$$
\sigma \times B R(i i \rightarrow H \rightarrow f f)=\frac{\sigma_{i i} \cdot \Gamma_{f f}}{\Gamma_{H}}
$$

where $\sigma_{i i}$ is the production cross section through the initial state $i i, \Gamma_{f f}$ the partial decay width into the final state $f f$ and $\Gamma_{H}$ the total width of the Higgs boson.

The leading order (LO) motivated scale factors $\kappa_{i}$ are defined in such a way that the cross sections $\sigma_{i i}$ and the partial decay widths $\Gamma_{i i}$ associated with the SM particle $i$ scale with the factor $\kappa_{i}^{2}$ when compared to the corresponding SM prediction. Taking the process $g g \rightarrow H \rightarrow \gamma \gamma$ as an example, one would use as cross section:

$$
(\sigma \cdot B R)_{(g g \rightarrow H \rightarrow \gamma \gamma)}=\sigma_{S M(g g \rightarrow H)} \cdot B R_{S M(H \rightarrow \gamma \gamma)} \cdot \frac{\kappa_{g}^{2} \cdot \kappa_{\gamma}^{2}}{\kappa_{H}^{2}}
$$

where the values and uncertainties for both $\sigma_{S M(g g \rightarrow H)}$ and $B R_{S M(H \rightarrow \gamma \gamma)}$ are taken from Refs. [3] for a given Higgs boson mass hypothesis.

The simplest model assumes that all couplings are modified by a single scaling parameter $\kappa$. In this case the fit to the data yields a value of $\kappa=1.19 \pm 0.11($ stat $) \pm$ 0.03 (syst) \pm 0.06 (theory) corresponding to the square root of the global signal strength $\mu$ shown in Figure 1 .

The relative couplings to fermions and bosons are tested in Section 5.1, assuming two common scale factors for these two sectors. The ratio of couplings to $\mathrm{W}$ and $\mathrm{Z}$ bosons, related to the custodial symmetry, is discussed in Section 5.2.1. The ratio of down to up quark type couplings, that is very interesting for several extensions of the $\mathrm{SM}$, is discussed in Section 5.2.2. The ratio of couplings to the lepton and quark sectors is given in Section 5.2.3. The possible effect of beyond SM particles on the indirect coupling to gluons and photons, that in the SM proceeds via loops and is particularly sensitive the new physics, is given in Section 5.3.
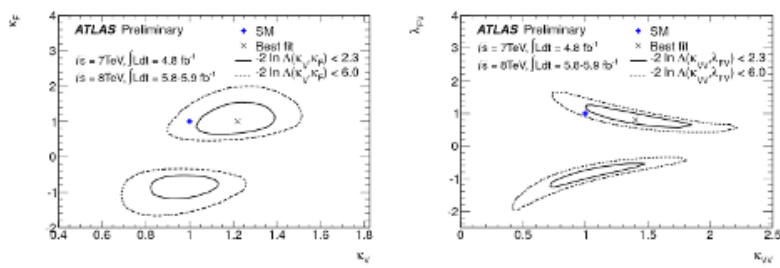

Figure 4. Fits for 2-parameter benchmark models probing different coupling strength scale factors for fermions and vector bosons, the left plot shows the correlation of the coupling scale factors, $\kappa_{F}$ and $\kappa_{V}$, assuming no non-SM contribution to the total width; The right plot shows the correlation of the ratio of coupling scale factors $\lambda_{F V}=\kappa_{F} / \kappa_{V}$ and $\kappa_{V V}=\kappa_{V} \cdot \kappa_{V} / \kappa_{H}$ without assumptions on the total width (Ref. [5]).

\subsection{Couplings to Fermions and Vector Bosons}

This benchmark is an extension of the single parameter $\mu$ fit, where a different strength for the fermion and vector coupling is probed. It assumes that only SM particles contribute to the $H \rightarrow \gamma \gamma$ and $g g \rightarrow H$ vertex loops. The fit is performed in two variants: with and without the assumption that the total width of the Higgs boson is given by the sum of the known SM Higgs boson decay modes (modified in strength by the appropriate fermion and vector coupling scale factors).

\subsubsection{Assuming only SM particles contribute to the total width}

The fit parameters are the coupling scale factors $\kappa_{F}=\kappa_{t}=$ $\kappa_{b}=\kappa_{\tau}$ for all fermions and $\kappa_{V}=\kappa_{W}=\kappa_{Z}$ for all vector couplings. The left plot of Figure 4 shows the result of the fit to this benchmark. Only the positive sign between $\kappa_{F}$ and $\kappa_{V}$ is physical and some sensitivity to this sign is gained from the negative interference between the W-loop and t-loop in the $H \rightarrow \gamma \gamma$ decay. The fit gives a small preference to the local minimum close the SM point. The $68 \%$ CL intervals of $\kappa_{F} \in[-1.0,-0.7] \cup[0.7,1.3]$ and $\kappa_{V} \in[0.9,1.0] \cup[1.1,1.3]$ when profiling over all other parameters. These intervals combine all experimental and theoretical systematic uncertainties. The $95 \% \mathrm{CL}$ intervals are $\kappa_{F} \in[-1.5,-0.5] \cup[0.5,1.7]$ and $\kappa_{V} \in[0.7,1.4]$. The compatibility of the SM hypothesis with the best fit point is $21 \%$.

\subsubsection{Relaxing the assumption on the total width}

Without the assumption on the total width, only ratios of coupling scale factors can be measured. Hence there are now the following free parameters, $\lambda_{F V}=\kappa_{F} / \kappa_{V}, \kappa_{V V}=$ $\kappa_{V} \cdot \kappa_{V} / \kappa_{H} \cdot \lambda_{F V}$ is the ratio of the fermion and vector coupling scale factors, and $\kappa_{V V}$ an overall scale that includes the total width and applies to all rates. The right plot of Figure 4 shows the results of this fit. The $68 \%$ and $95 \%$ CL intervals of $\lambda_{F V}$ when profiling over $\kappa_{V V}$ are, $\lambda_{F V} \in$ $[-1.1,-0.7] \cup[0.6,1.1]$ and $\lambda_{F V} \in[-1.8,-0.5] \cup[0.5,1.5]$, respectively. The $2 \mathrm{D}$ compatibility of the SM hypothesis with the best fit point is $21 \%$. It should be noted that the assumption on the total width gives a strong constraint on the 


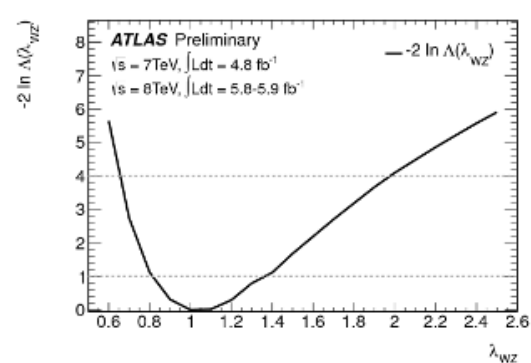

Figure 5. Fit to benchmark model probing for deviations between $\mathrm{W}$ and $\mathrm{Z}$ vector boson couplings using the ratio $\lambda_{W Z}=$ $\kappa_{W} / \kappa_{Z}$ (Ref. [5]).

fermion coupling scale factor $\kappa_{F}$, since it is dominated by the b-decay width, which is currently not well constrained by data. The measurement of $\kappa_{V V}$, profiling the $\lambda_{F V}$ parameter yields: $\kappa_{V V}=1.2_{-0.6}^{+0.3}$.

The value of the scale factor affecting the coupling of the new particle to gauge bosons, $\kappa_{V}$, is both directly and indirectly constrained by several channels. The coupling to fermions is directly observed in the $H \rightarrow \tau^{+} \tau^{-}$and $H \rightarrow$ $b \bar{b}$ channels, the direct constraint is very weak as these signals don't yet show sensitivity to the SM Higgs boson. A value of $\kappa_{F}$ significantly deviating from zero is indirectly observed through the constraints from channels searching for both the $g g \rightarrow H$ and VBF production processes.

\subsection{Probing the structure of Fermion and Vector Couplings}

Both previous benchmarks assume that the coupling scale factors for the W- and Z-boson are identical $\left(\kappa_{V}=\kappa_{W}=\right.$ $\left.\kappa_{Z}\right)$ and that the scale factors for all fermions are identical $\left(\kappa_{F}=\kappa_{t}=\kappa_{b}=\kappa_{t}\right)$. In the following subsections both assumptions are relaxed and tested. Since this test is focused on coupling ratios the assumption on the total width has been relaxed.

\subsubsection{Probing the custodial symmetry of the $W$ and $Z$ couplings}

Identical coupling scale factors for the $\mathrm{W}$ - and Z-boson are required within tight bounds by $\mathrm{SU}(2)_{V}$ custodial symmetry and the $\rho$ parameter measurements at LEP [7]. To test this directly in the Higgs sector, the ratio $\lambda_{W Z}=\kappa_{W} / \kappa_{Z}$ is probed. The same assumptions as in Section 5.1.2 on $\kappa_{F}$ are made $\left(\kappa_{F}=\kappa_{t}=\kappa_{b}=\kappa_{\tau}\right)$. The free parameters are $\kappa_{Z Z}=\kappa_{Z} \cdot \kappa_{Z} / \kappa_{H}, \lambda_{W Z}=\kappa_{W} / \kappa_{Z}$ and $\lambda_{F Z}=\kappa_{F} / \kappa_{Z}$.

Figure 5 shows the likelihood distribution for the ratio $\lambda_{W Z}$ when profiling the $\kappa_{Z Z}$ and $\lambda_{F Z} \cdot \lambda_{W Z}=1.07_{-0.27}^{+0.35}$ is obtained at $68 \% \mathrm{CL}$. Fits to the other two parameters of the model yields $\kappa_{Z Z}=1.3_{-0.6}^{+0.9}$ and $\lambda_{F Z} \in[-1.1,-0.5] \cup$ $[0.6,1.2]$ at $68 \% \mathrm{CL}$. The 3D compatibility of the SM hypothesis with the best fit point is $33 \%$.

\subsubsection{Probing the up- and down-type fermion symmetry}

In many extensions of the SM, the couplings of the light Higgs boson to up-type and down-type fermions differ.

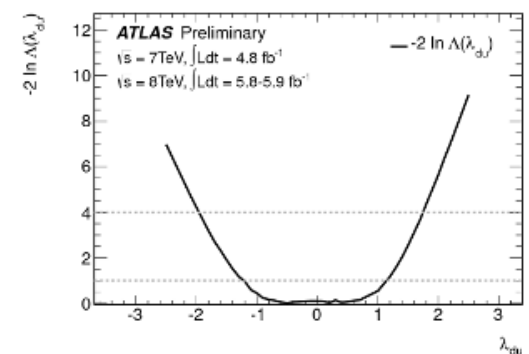

Figure 6. Fit to benchmark model probing for deviations between up-type and down-type fermion symmetry using ratio $\lambda_{d u}=\kappa_{d} / \kappa_{u}$ (Ref. [5]).

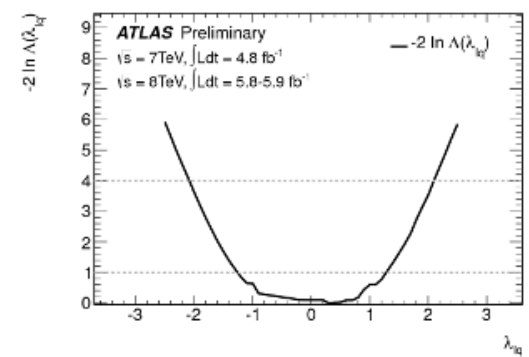

Figure 7. Fit to benchmark model probing for deviations between lepton and quark fermion symmetry using ratio $\lambda_{l q}=\kappa_{l} / \kappa_{q}$ (Ref. [5]).

The free parameters are, $\kappa_{u u}=\kappa_{u} \cdot \kappa_{u} / \kappa_{H}, \lambda_{d u}=\kappa_{d} / \kappa_{u}$ and $\lambda_{V u}=\kappa_{V} / \kappa_{u}$. Figure 6 shows the likelihood distribution for the ratio $\lambda_{d u}=\kappa_{d} / \kappa_{u}$. The $68 \%$ and $95 \% \mathrm{CL}$ intervals of $\lambda_{d u}$ when profiling the $\kappa_{u u}$ and $\lambda_{V u}$ parameters are $\lambda_{d u} \in[-1.2,1.2]$ and $\lambda_{d u} \in[-2.0,1.8]$, respectively. The range in the measurement of $\lambda_{d u}$ is dominated by the low sensitivity in the $H \rightarrow \tau^{+} \tau^{-}$and $H \rightarrow b \bar{b}$ channels. The 3D compatibility of the SM hypothesis with the best fit point is $33 \%$. Fits to the additional parameters yield $\kappa_{u u}=1.0_{-0.3}^{+0.4}$ and $\lambda_{V u} \in[-1.3,-1.1] \cup[0.8,1.6]$ at $68 \%$ CL.

\subsubsection{Probing the quark and lepton symmetry}

In summary, the ratio $\kappa_{l} / \kappa_{q}$ between leptons and quarks is probed. The same assumptions are made on $\kappa_{V}=\kappa_{W}=$ $\kappa_{Z}, \kappa_{l}=\kappa_{\tau}$ and $\kappa_{q}=\kappa_{b}=\kappa_{t}$. The free parameters are, $\kappa_{q q}=\kappa_{q} \cdot \kappa_{q} / \kappa_{H}, \lambda_{l q}=\kappa_{l} / \kappa_{q}$ and $\lambda_{V q}=\kappa_{V} / \kappa_{q}$. Figure 7 shows the likelihood distribution for the ratio $\lambda_{l q}=\kappa_{l} / \kappa_{q}$. The $68 \%$ and $95 \%$ CL intervals of $\lambda_{l q}$ when profiling the $\kappa_{q q}$ and $\lambda_{V q}$ observables are $\lambda_{l q} \in[-1.3,1.3]$ and $\lambda_{l q} \in$ $[-2.1,2.1]$, respectively. The range of $\lambda_{l q}$ is dominated by the low sensitivity in the $H \rightarrow \tau^{+} \tau^{-}$channel. The 3D compatibility of the SM hypothesis with the best fit point is $31 \%$. Fits to the additional parameters yield: $\kappa_{q q}=1.0_{-0.4}^{+0.5}$ and $\lambda_{V q}=1.1_{-0.3}^{+0.6}$.

The ratio $\lambda_{W Z}$ probes the custodial symmetry through the relative coupling of the new particle to the $W$ and $\mathrm{Z}$ bosons. The fit of $\lambda_{W Z}$ is in part directly constrained by the direct decays in the $H \rightarrow W W^{(*)} \rightarrow \ell v \ell v$ and $H \rightarrow Z Z^{(*)} \rightarrow 4 \ell$ channels. The $\kappa_{W}$ part is also indirectly constrained by the $H \rightarrow \gamma \gamma$ channel since the decay branching ratio gets a dominant contribution from $\kappa_{W}$. The measured value of $\lambda_{W Z}$ is in agreement, within the uncertainties (which are at present dominated by statistical un- 


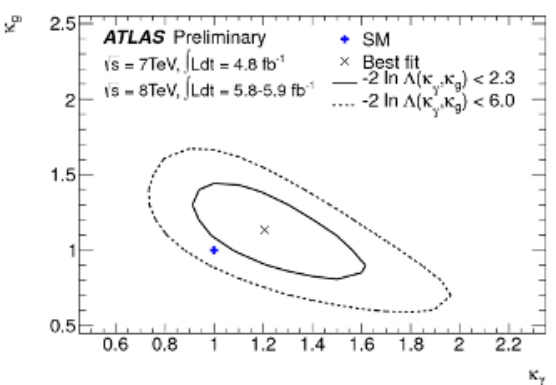

Figure 8. Fit to benchmark model probing for contributions from non-SM particles based on $g g \rightarrow H$ and $H \rightarrow \gamma \gamma$ loops assuming no extra contribution to the total width (Ref. [5]).

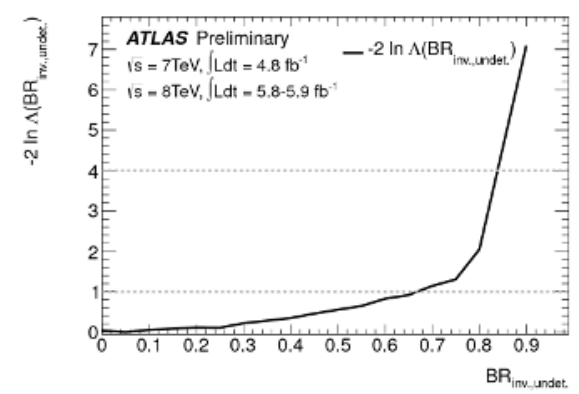

Figure 9. Fit to benchmark model probing for contributions from non-SM particles based on $g g \rightarrow H$ and $H \rightarrow \gamma \gamma$ loops assuming a possible invisible or undetectable branching ratio $B R_{\text {int.,undet. }}$. (Ref. [5]).

certainties) with the custodial symmetry, as expected for a SM Higgs boson.

The uncertainty on the measurement of the relative strength of the coupling of the observed state to the upand down-type fermions is dominated by the measurement of its decay in the $H \rightarrow \tau^{+} \tau^{-}$and $H \rightarrow b \bar{b}$ channels. The ratio $\lambda_{d u}$ is only weakly constrained by the current data. Similarly the $\lambda_{l q}$ ratio mainly relies on the $H \rightarrow \tau^{+} \tau^{-}$ channel and is only weakly constrained.

\subsection{Probing Potential Non-SM Particle Contributions}

This case allows for new particle contributions either in loops or in new final states. All coupling scale factors of known $\mathrm{SM}$ particles are as in the SM, i.e. $\kappa_{i}=1$. For the $H \rightarrow \gamma \gamma$ and $g g \rightarrow H$ vertices, effective scale factors $\kappa_{g}$ and $\kappa_{\gamma}$ are introduced. They allow for extra contributions from new particles. The potential new particles contributing to the $H \rightarrow \gamma \gamma$ and $g g \rightarrow H$ loops, may or may not contribute to the total width of the observed state from direct invisible decays or decays into event topologies that are not distinct from the background. In the latter case the resulting variation in total width is parameterized in terms of the additional branching ratio $B R_{\text {inv.,undet }}$.

Assumed that there are no sizeable extra contributions to the total width caused by the non-SM particles. The free parameters are $\kappa_{g}$ and $\kappa_{\gamma}$, the $68 \%$ and $95 \%$ CL contours are shown in Figure 8. The best fit values and uncertainties are $\kappa_{g}=1.1_{-0.3}^{+0.2}$ and $\kappa_{\gamma}=1.2_{-0.2}^{+0.3}$ at $68 \%$ CL. The 2D compatibility of the SM hypothesis with the best fit point is $18 \%$.

By constraining some of the factors to be equal to their SM values, it is possible to probe for new non-SM decay modes that might appear as invisible or undetectable final states. The free prameters are $\kappa_{g}, \kappa_{\gamma}$ and $B R_{\text {inv.,undet. }}$. In this model, the modification to the total width is parametrized as follows:

$$
\Gamma_{H}=\frac{\kappa_{H}^{2}\left(\kappa_{i}\right)}{\left(1-B R_{\text {inv.,undet. }}\right)} \Gamma_{H}^{S M}
$$

Figure 9 shows the likelihood as a function of $B R_{\text {inv.,undet. }}$ when $\kappa_{g}$ and $\kappa_{\gamma}$ are profiled. The best fit values and uncertainties at $68 \% \mathrm{CL}$ are $\kappa_{g}=1.1_{-0.2}^{+1.4}, \kappa_{\gamma}=1.2_{-0.2}^{+0.3}$ and $B R_{\text {inv. }{ }_{\text {,undet }}}<0.68$. The 3D compatibility of the SM hypothesis with the best fit point is $35 \%$.

\section{Conclusion}

Using data taken in 2011 and 2012, at centre-of-mass energies of respectively $7 \mathrm{TeV}$ and $8 \mathrm{TeV}$ and corresponding altogether to approximately $10 \mathrm{fb}^{-1}$, the ATLAS collaboration has reported the observation of a new particle with a mass of $m_{H}=126.0 \pm 0.4($ stat $) \pm 0.4$ (syst) $\mathrm{GeV}$, in the search for the Standard Model Higgs boson. The same data set was further analysed to investigate the coupling properties of the new observed particle. Simplified benchmark models were used to study the correlations of production and decay modes in different final states, and compare them to the predictions for the SM Higgs boson. Within the current statistical uncertainties and assumptions, no significant deviations from the Standard Model couplings are observed.

\section{References}

[1] ATLAS Collaboration, Phys. Lett. B716, 1-29 (2012)

[2] CMS Collaboration, Phys. Lett. B716, 30-61 (2012)

[3] LHC Higgs Cross Section Working Group, arXiv:1209.0040, arXiv:1201.3084 (2012)

[4] G. Cowan et.al., Eur. Phys. J. C71 1554 (2011)

[5] ATLAS Collaboration, Phys. Rev. D86 032003 (2012), ATLAS-CONF-2012-127

[6] LHCHXSWG-2012-001.pdf, https://twiki.cern.ch/twiki/bin/view/LHCPhysics

[7] CERN-PH-EP/2010-095, arXiv:1012.2367 (2010).

[8] ATLAS Collaboration, ATLAS-CONF-2012-162 